\title{
A SELF-ROUTING PHOTONIC SWITCH USING AN OPTICAL CONTENT ADDRESSABLE MEMORY
}

\author{
Subrat Kar and A. Selvarajan, \\ Department of Electrical Communication Engineering, \\ Indian Institute of Science, \\ Bangalore 560012
}

\begin{abstract}
A novel self-routed slot-switching scheme using an integrated optic directional cou ler is outlined using a pulse interval coded optical content adressuble memory (PICOCAM). The integrated optic devices required for its implementation are also outlined. This bit-switching approach avoids the bottleneck in speed imposed by the electronic drive circuitry in electrooptic switches.
\end{abstract}

\section{INTRODUCTION :}

Photonic switching has motivated research due to two needs firstly, the need for an ultra-fast switching technology and secondly, for a common technology for the transmission and switching of photonic data streams. The significant inroads made by the optical fiber into high-capacity data highways provide incentives for the switching of data sreams in the optical domain and the development of photonic switching architectures (PSAs). At ultra-fast data rates, it is only in the optical domain that the requisite data-rate transparency can be achieved.

While optical implementation remains the technology for the implementation of these photonic switches, usually the first step is a straight replacement of electronic switches by optical ones, such that the electrical overlay network processes the stripped headers and sets the homologous optical switch. In this approach, an electrical-to-optical conversion is implicit.

At multi-gigabaud rates, the overhead of optical-electrical- optical conversion and the limitation imposed by the electronic drive circuitry is unacceptable. Self-routing, in which the photonic data streams carry their own routing information and the bulk of the header processing is achieved by the photonic circuitry, addresses this problem and allows the transmission and switching of data at the full capacity of the optical medium. Moreover, self-routing offloads the amount of processing required from each node to the inputs where the relatively low-bandwidth electronics is adequate.

In this paper, we propose a scheme for bit-wise self-routing in a $2 \times 2$ photonic switching element (PSE) - an integrated optic directional coupler (IODC) as shown in Fig. 1.

\section{THE INTEGRATED OPTIC DIRECTIONAL COUPLER}

he IODC, in its most general form, consists of two integrated optical waveguides running parallel to each other over a coupling length $L_{c}$. In this configuration, the gap between the waveguides is such that the evanescent tail of the field in one waveguide extends into the other, giving rise to a coupling coefficient $\kappa$. Depending on the coupling length, light (of a particular wavelength) input to one waveguide of the IODC either crosses over to the other waveguide (or stays in the same waveguide). If the light crosses over in the default state, an electric field may be applied to an overlying electrode to change the waveguide refractive index so that the light couples back to the same waveguide.
Thus the PSE achieves one of two states - through or cross. The power transfer efficiency, $\eta$, may be expressed as

$$
\eta=\frac{\sin ^{2}\left[\kappa \mathrm{L}\left(1+(\delta / \kappa)^{2}\right)\right.}{1+(\delta / \kappa)^{2}}
$$

where the coupling coefficient $\kappa=\Delta \beta / 2$

Functionally, the IODC can be thought of as a two-input, two-output two-state device (all the states of a generalized four-port device, like the upper and lower broadcast are not used). As a logic element, the IODC PSE may be described by the boolean expression $E=D=A . C+B . C$, where $A, B, C$ and $D$ are the two inputs and two outputs respectively and $C$ is the control signal.

\section{PRINCIPLE:}

Since the coupling of light from one waveguide to another depends on the wavelength of the light, for a given coupling length Lc, we can find two wavelengths $\lambda_{1}$ and $\lambda_{2}$ [ Table.1]

$\begin{array}{llll}\kappa & 0.05819 & 0.0389056 & 0.0558075 \\ \mathrm{~L} & 26.99268 & 40.37453 & 28.14 \\ \Lambda_{1} & 1.3 & 0.8230 & 1.30 \\ \Lambda 2 & 0.63 & 1.2989 & 0.63\end{array}$

Table.1

such that light input on wavelength $\lambda_{1}$ would cross over to the other waveguide while light input on wavelength $\lambda_{2}$ would remain in the same waveguide. Therefore the two-state functionality of the switch is preserved - with the two states, cross and through, being achieved by presenting the data on two different fixed wavelengths. Since this avoids the associated drive circuitry, the scheme is elegant and allows data throughput at near optical rates. Further the switching scheme is inherently real-time i.e. the response of the switch is less than the data bit width. the responsibility of presenting the data to the switch now devolves on the input - thus the data must be recognized and converted to the appropriate wavelength before it is input to the switch.

We assert that, in any non-faulty rearrangeably non-blocking PSA, if the routing architecture is known, the set of connections $\zeta$ between any set of inputs and outputs is deterministic. Further, this assertion implies that if $I_{n}$ is the set of inputs and $O_{n}$ is the set of outputs, then for all $\zeta \varepsilon\left\{I_{n} \times O_{n}\right\}$, the state $\sigma_{n}$ of every switch $n$ is known.

Example: Consider a $3 \times 3$ half-square matrix as in Fig.2(a). The connections that can be thought of are a set of ordered pairs $(x, y)$ such that $x \in I$ and $y \in O$ and $\left\{(x, y) \in\left\{I_{n} X O_{n}\right\}\right\}$ where $I$ is the set of inputs and $O$ the set of output ports. Letting $n(I)$ denote the cardinality of $I$, the number of connections that can be made is 
${ }^{\mathrm{n}(\mathrm{I})} \mathbf{P} \mathbf{n}(\mathrm{O})$. For this half-square matrix, we can easily enumerate the elements of $\left\{I_{n} \times O_{n}\right\}$ as (assuming $I=\{10, i 1, i 2\}$ and $\left.\mathrm{O}=\left\{\mathrm{Oo}_{0}, \mathrm{O}_{1}, \mathrm{O}_{2}\right\}\right)\left\{\left(\mathrm{i}_{0}, \mathrm{O}_{0}\right),\left(\mathrm{i}_{0}, \mathrm{O}_{1}\right),\left(\mathrm{i}_{0}, \mathrm{O}_{2}\right),\left(\mathrm{i}_{1}, \mathrm{O}_{0}\right),\left(\mathrm{i}_{1}, \mathrm{O}_{1}\right)\right.$, $\left.(\mathrm{i} 1, \mathrm{O} 2),(\mathrm{i} 2, \mathrm{Oo}),\left(\mathrm{i} 2, \mathrm{O}_{1}\right),\left(\mathrm{i}_{2}, \mathrm{O}_{2}\right)\right\}$. The number of possible connections are given by the following six sets: $S_{1}=(0,0),(1,1),(2,2) ; S_{2}$ $=(0,1),(1,0),(2,2) ; S_{3}=(0,2),(1,0),(2,1) ; S_{4}=(0,0),(1,2),(2,1)$; S5 $=(0,1),(1,2),(2,0) ; S_{6}=(0,2),(1,1),(2,0)$.

We can see that, for some connections ( represented by the set ST ), the switch is in the through state while, for other connections ( represented by the set $S_{X}$ ), it is in the cross state. Furthermore, for each switch, the sets $S_{T}$ and $S_{X}$ are predetermined and $S_{T}=$ $\left\{I_{n} X O_{n}\right\} \backslash S X$.i.e. $S_{X}$ is the complement of $S_{T}$ in the universe of $\left\{I_{n} X O_{n}\right\}$. In switch 1 , for instance, $S_{T}=\left\{S_{1}, S_{2}, S_{3}, S_{4}\right\}$ and $\mathrm{SX}=\left\{\mathrm{S}_{5}, \mathrm{~S}_{6}\right\}$.

$\begin{array}{lll}\text { S.No. } & \text { ST } & \text { Sx } \\ 1 & 1,2,3,4 & 5,6 \\ 2 & 1,4 & 2,3,5,6 \\ 3 & 1,2,5 & 3,4,6\end{array}$

Table.2

3.THE OPTICAL CONTENT ADDRESSABLE MEMORY SCHEME:

The content addressable scheme should give an output of 1 if it contains the required data and $0($ null $)$ if it does not. To implement an optical element with this functionality (hereafter referred as CAM ), we propose the following scheme.

The recognition of the data at the switch is done using a pulse-interval-coded optical content addressable memory (PICOCAM). The set of possible destinations D ( e.g. two destinations for a single switch ) of a data bit is split into two sets ST and SX as shown in Table I. A data bit bound for a destination $\mathrm{d} \varepsilon \mathrm{ST}$ (Case I) requires the PSE to be in the through state and must be presented on wavelength $\lambda_{1}$. Similarly, a data bit going to a destination $\mathrm{d} \varepsilon \mathrm{SX}$ (Case II) requires the PSE to be in the cross state and must be presented on a wavelength $\lambda_{2}$. Each data bit is encoded in the PICOCAM format as follows : each bit is translated into an equivalent header consisting of $n+l$ slots $\left(\mathrm{S}_{\mathrm{n}}\right)$ of duration $\mathrm{T}$ each where $n$ is the number of destinations with a guard delay $E$ between each slot (for a single switch, $n=2$ ) as shown in Fig.3.

We take an optical fiber with $\mathrm{n}$ taps in it at integral multiples of length d [Fig.4(a)-(b)] where $\mathrm{d}<$ ( $2 \mathrm{x}$ data-bit width) and $\mathrm{n} \in \mathrm{I}$. We input two pulses at either end of the fiber. One of these pulses serves as the reference pulse ( $R_{p}$ ) and the other as the addressing pulse $\left(A_{p}\right)$. The intersection point of these two pulses is used to trigger a APD-LD combination. The time delay between the two pulses is critical to the addressing scheme. Let the time taken by a pulse to travel a distance $d$ be $r$. Then if the tap 6 is to be addressed, $R_{p}$ must be at tap 5 when $A_{p}$ is fed in .i.e. the time delay between the two pulses is $5 \tau$. If tap 4 is to be addressed, then $R_{p}$ must be at tap 1 when $A_{p}$ is fed in .i.e. the time delay between the $R_{p}$ and the $A_{p}$ is given by $\tau$ or,equivalently, $((2 \times 4)-(6+1)) r$. In general, if the $Z$ th tap is to be addressed, the time delay between the two pulses is given by

$$
\tau(2 \mathrm{Z}-\mathrm{n}-1)
$$

This is easily verified for the two cases described above. Putting $\tau=6$ and $\tau=4$, we obtain the required time delays as $5 \tau$ and $t$ respectively. The expression (1) is negative for $\tau<(n+1) / 2$.i.e., the position of $R_{p}$ and $A_{p}$ are reversed for this condition where the tap to be addressed is either 1,2 or 3 . Since this does not agree with our previously outlined scheme for the header, we must modify the above scheme such that $R_{p}$ always is ahead of the $A_{p}$. We add the term $2(n+1)$ to $\tau$ - physically, this is equivalent to adding $2 d(n+1)$ unit lengths of fiber to the right hand side of the CAM. The revised expression for $\tau$ is given by

$$
\begin{gathered}
\tau(2 Z-(n+1)+2(n+1)) \\
\tau(2 Z+n+1)
\end{gathered}
$$

which is always positive, implying that the relative positions of the $\mathbf{R}_{\mathrm{p}}$ and the $\mathrm{A}_{\mathrm{p}}$ are always unchanged. The trade-off incurred is that the increase in the time delay implies an increase in the header maximal length and a consequent decrease in the data rate.

In general, the pulses $R_{p}$ and $A_{p}$ would arrive serially on one fiber. Thus the input to the two ends of the fiber will, in general, be derived from a passive splitter stage as shown in the figure. Thus, when the $R_{p}$ pulse arrives at the point $A$, it suffers a split $(3 \mathrm{~dB})$ into two identical pulses $R_{p 1}$ and $R_{p 2}$ that start propagating towards points $B$ and $C$ in opposite directions. The pulses $R_{p 1}$ and $R_{p 2}$ meet at the midpoint $D$. Thus, it must follow that there must be no tap at the midpoint $D$ as this tap would get selected for all data bits .i.e. the taps must be spaced $d$ units of length apart but asymmetric about the point $D$.

Since the pulses $R_{p}$ and $A_{p}$ both split into two counter-propagating pulses (denoted by $R_{p 1}, R_{p 2}$ and $A_{p 1}, A_{p 2}$ respectively), it follows from arguments of symmetry that the two pairs of pulses $\left(R_{p 1}, A_{p 2}\right.$ ) and ( $\left.R_{p 2}, A_{p 1}\right)$ meet at two points - denoted by X1 and X2exactly symmetric about the midpoint $D$. Thus with the scheme outlined to this point, the taps at $X 1$ and $X 2$ get selected .i.e. the tap addressing scheme is not unique. If we are to address an unique tap with the $R_{p}$ and $A_{p}$, we must remove one of these points either X1 or X2. A simple way of doing this is to merely double the length of the fiber $\mathrm{BC}$ and place all taps on side of the new midpoint (and none $O N$ the midpoint ). Therefore, the time delay between the $R_{p}$ and $A_{p}$ is again doubled and the data rate halved but the tap addressed is unique. We have thus introduced two modifications. Firstly, we made the tap positions asymmetric about the midpcint $D$ - this modification, however, does not alter expression (1). Sec. 'ly, we have doubled the fiber length (BC) - therefore a multip. ative factor of two will be added to (1) which becomes

$$
2 T(2 Z-n-1)
$$

We have assumed that a tap consists of a splice at a single point. However, with two optical pulses moving in opposite directions, the tap must appear as shown in the adjacent figure. Assuming that the point $G$ (or $G^{\prime}$ ) in Figs.4(a) and (b) are the same, it is obvious that the original expression for the delay between $R_{p}$ and $A_{p}$ must be reduced by $2 f$ where $f$ is the time taken to travel a distance $k$, as marked in Fig.4(b). This can be seen from the fact that $A_{p}$ ( or $A_{p 1}$ ] ) now reaches the tap point $\mathrm{H} 2 \mathrm{f}$ units of time sooner. Expression (1) now becomes

$$
2(T(2 Z-n-1)-f)
$$

Implementation of a complete ring would waste real estate on the necessary bends- they would be limited by the bending radius required to achieve the minimum acceptable bending loss. It has been demonstrated, both theoretically [1] and experimentally [2], that bend radii smaller than $1 \mathrm{~cm}$ lead to unacceptably high leakage losses. Even though curved bends are usually considered, $180^{\circ}$ bends may be achieved by using halved 3-stage $\Delta \beta$-reversal directional couplers witiı metallized mirrors at one end [3] or with 
etched pit mirrors. Given the typical 1000:1 length-width ratio of the directional coupler, this achieves a very economical use of a given substrate area, either quadratic or otherwise. Typically, the reversal element would have an overall length of around $1.5 \mathrm{~mm}$ and a width of around $0.2 \mathrm{~mm}$ ( mainly due to the width of the electrodes which are required for fine-tuning the coupling length changes due to post-diffusion polishing of the reflecting surface). With this form of integrated optic implementation, the combination of $R_{p 1}$ and $A_{p 2}$ are of no consequence as, even though they occur, they do not trigger any taps as argued previously from symmetricity considerations. The doubling of the fiber length is consequently, unnecessary and the data rate is doubled.

The first slot $S_{0}$ is the reference pulse $\left(R_{p}\right)$ and is always 1 . denoting the beginning of the header while $\mathrm{S}_{1}$ (in general $\mathrm{Si}$, $\mathrm{i}=2 . \mathrm{N}$ ) is called the address pulse (Ap). If the destination of the data bit is denoted by $d$, then $S_{1}=1$ if $d$ is in $S x$ and $S_{2}=1$ if $d$ is in ST. The content addressable memory is implemented by fabricating a folded-ring integrated optic waveguide with a passive $Y$-splitter at one end and a halved 3-stage $\Delta \beta$-reversal directional coupler with two pairs of Y-branched counter-taps as shown in Fig.5(a). Two of the $\mathrm{Y}$-branches $\left(\mathrm{Y}_{\mathrm{c} 1}\right.$ and $\left.\mathrm{Y}_{\mathrm{c} 2}\right)$ tap optical power from pulses travelling in the clockwise direction while the other two Y-branches ( $\mathrm{Yac1}_{\mathrm{ac}}$ and $\mathrm{Y}_{\mathrm{ac2}}$ ) tap optical power from pulses travelling in the anticlockwise direction. The suggested integrated optic realisation suggested by us is as shown in Fig.5(b). $Y_{\mathrm{cl}}$ and $Y_{\text {ac1 }}$ lead to a avalanche photo-diode-laser diode pair (APD-LD ${ }_{1}$ ) which emits light at wavelength $\lambda_{1}$ while $Y_{\mathrm{c} 2}$ and $\mathrm{Y}_{\mathrm{ac} 2}$ lead to APD-LD 2 which emits light at wavelength $\lambda_{2}$.

The header is input at the passive Y-splitter end of the PICOCAM structure. The pulses $R_{p}$ and, after a delay, Ap split into two counter-propagating pulses each - denoted as $\left(\mathbf{R}_{\mathbf{p} 1}, \mathbf{R}_{\mathbf{p} 2}\right)$ and ( $A_{\mathbf{p} 1}$, $\left.A_{p 2}\right)$. The meeting point $P$ of two of these pulses $-R_{p 1}$ and $A_{p 2}-$ and the consequent summation of optical power is the addressing scheme. We show that if the delay between $R_{p 1}$ and $A_{p 2}$ is equal to $T(2 z+n+1)$, the $z^{\text {th }}$ APD-LD combination is triggered. Thus by appropriately adjusting the delay between $R_{p 1}$ and $A_{p 1}$, we may address any of the two APD-LD combinations and output one data pulse on the desired wavelength - either $\lambda_{1}$ or $\lambda_{2}$.The complete self-routing schematic is shown in Fig.7.

In implementing the PICOCAM scheme, there would be an inherent insertion loss and splitting loss. The insertion loss depends on the lengths of fiber inputs, the waveguide propagation loss and the switching element loss ( due to material absorbtion, scattering, incorrect coupling losses between fiber and substrate ). However, in our scheme, the threshold detection trigger serves to regenerate the pulse and compensates for the insertion loss. Closed form expressions for the worst case insertion loss may be derived as follows. If $S$ is the power splitting ratio at each tap, this implies that the loss at the each Y-splitter is

$$
10 . \log _{10}\left(P_{i} /\left(P_{i}-\text { S.Pi }\right)\right)
$$

If there are $\mathrm{n}$ taps, the total splitting loss at the $\mathrm{m}^{\text {th }}$ tap is given by

$$
2 . L_{c}+m .10 \log 10[1 /(1-S)]+L_{j}
$$

where $\mathrm{L}_{c}=$ connector loss and $\mathrm{L}_{\mathrm{j}}=$ junction loss

The value of the insertion loss vs number of taps for splitting ratios ranging from 0.1 to 1 is shown in Fig.6. For an insertion loss of 15 $\mathrm{dB}$, a splitting ratio of 0.1 would allow six taps. With an allowed wafer diameter of $3^{\prime \prime}$, the maximum propagation delay of an optical pulse is $\mathbf{0 . 5 0 6 8 5}$ nanoseconds. Thus if there are $n$ taps, the mini- mum delay is $(0.50685 / \mathrm{N})$ ns. For $\mathrm{N}=6$, the tap spacing is, equivalently, 84.4 picoseconds. The data rate, therefore, is determined by the largest pulse interval i.e. 506.4 picoseconds - which corresponds to a fixed data throughput of $1.97 \times 10^{9} \mathrm{~Hz}$ or nearly 2 GHz.

4. CONCLUSIONS :

We have outlined a novel self-routing scheme with a content addressed optical memory and a pulse position addressed header. However, the scheme only allows data to be throughput at a rate fixed by the tap spacing and therefore is not suitable for data arriving at different rates. The responsibility of presenting the data in the suitable format, moreover, lies with the source of the data. The proposal lends itself to an integrated optic implementation fairly easily. Several novel integrated optic elements have been proposed whose implementation is straight-forward. Since this routing scheme requires no switching in the electronic domain, the throughput expected is high and at the speeds defined largely by the optical inputs and the response times of the APD-LD combination.

\section{REFERENCES}

[1] E.A.J.Marcatili, Bell.Syst.Tech.Journal, 48 (1969) 2103.

[2] L.D.Hutcheson, I.A.White and J.J.Burke : "Losses in diffused $\mathrm{LiNbO}_{3}$ waveguides by directional changes", in Diaest of Topical Meeting on Integrated and Guided-wave optics. Incline Village, January 1980, paper WB2.

[3] K.H.Tietgen, R.Th.Kersten: “ $180^{\circ}$-turns in integrated op tics", Optics Communications, Vol.36, No.4, 15 Feb, 1981, pp.281284.

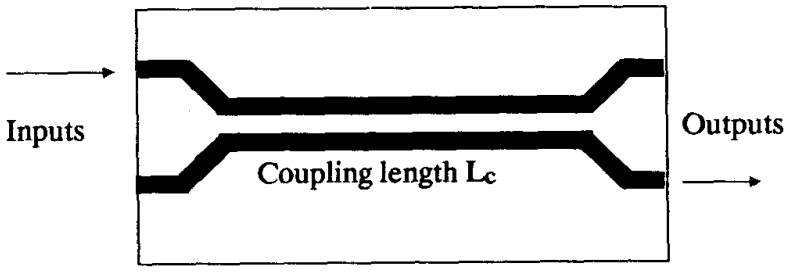

Fi.g.1: An integrated optic directional coupler

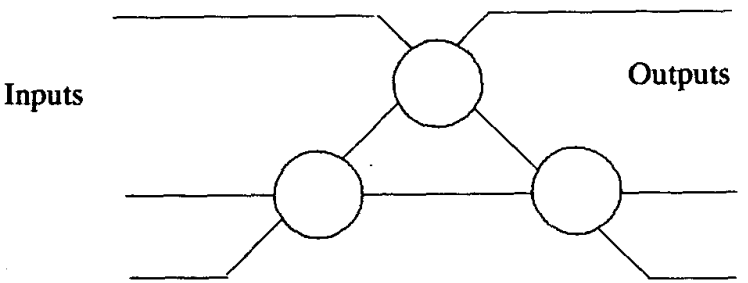

Fig.2: A $3 \times 3$ half-square matrix 


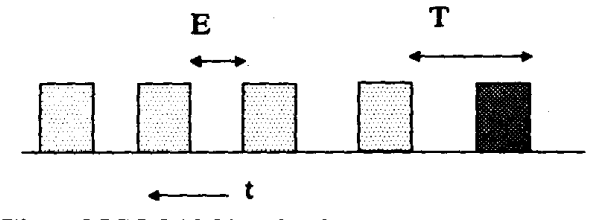

Fig.3 : PICOCAM header format

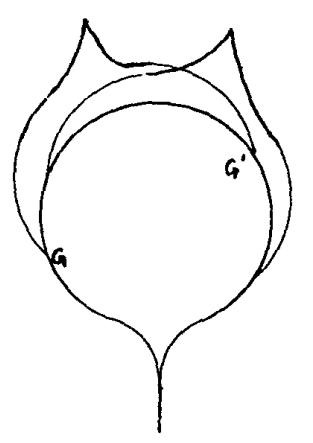

Fig.5(a) CAM implementation with folded-ring waveguide, passive $Y$-splitter and a halved 3-stage directional coupler

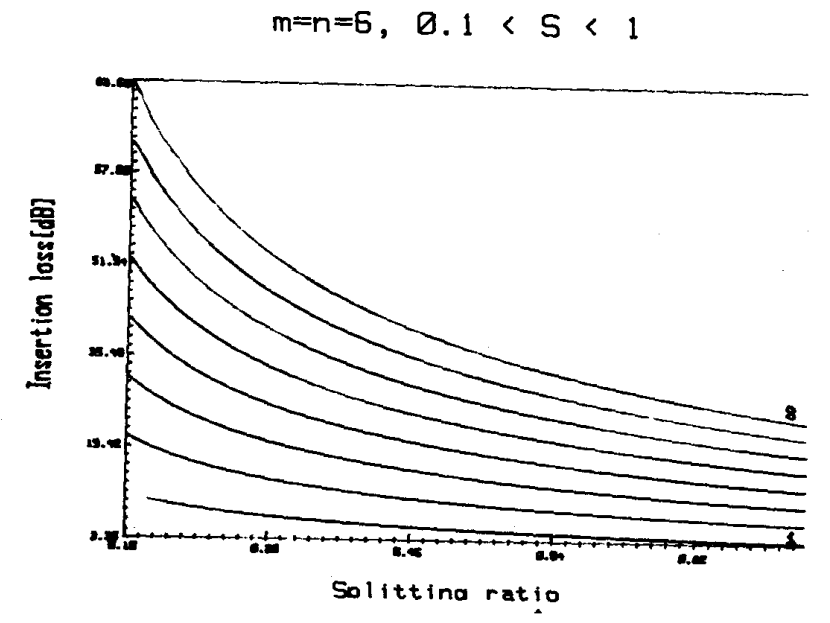

Fig.6: Insertion loss vs Number of taps with splitting ratios ranging from 0.1 to 1

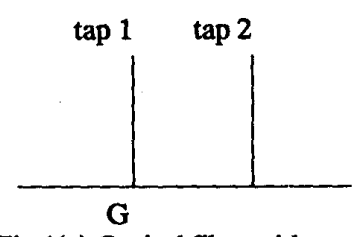

Fig.4(a) Optical fiber with n taps at integral multiples of length $d$

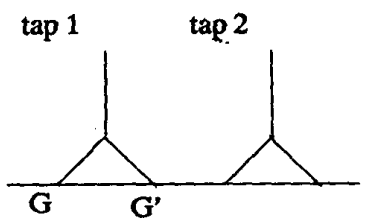

(b) directional tapping of the signal in the optical fiber

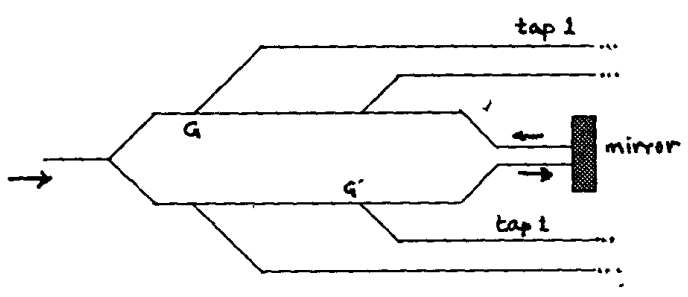

Fig.5(b) Integrated optic realisation of the CAM

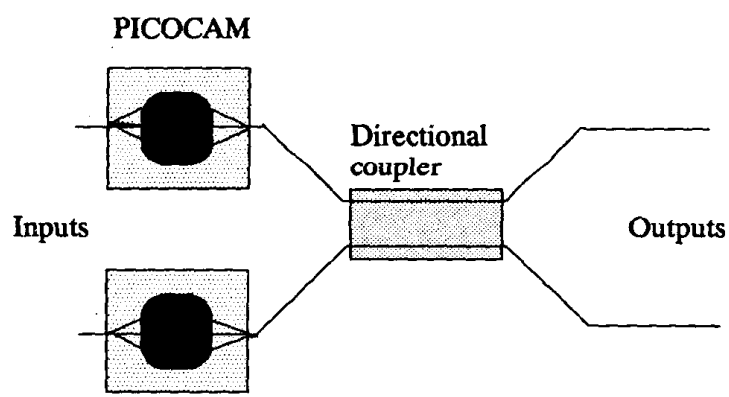

Fig. 7 : The self-routing scheme using two PICOCAM elements and an IODC 